\title{
Lichen planus occurring on radiotherapy site: a case report
}

\author{
Imene Ben Lagha, Sana Mokni, Amina Aounallah, Marwa Guerfala, Wafa Saidi, Lobna \\ Boussofara, Colandane Belajouza, Mohamed Denguezli, Rafiaa Nouira
}

Dermatology department, Farhat Hached University Hospital of Sousse, Tunisia

Corresponding author: Dr. Imene Ben Lagha, E-mail: imenebenlagha@gmail.com

\begin{abstract}
Lichen planus (LP) is an inflammatory skin condition characterized by the presence of pruritic, polygonal, purple flattopped papules and plaques typically symmetrically distributed. The occurrence of these lesions could be attributed to the isomorphic response of koebner occuring regularly in LP. A 38-year-old woman developed pruritic purplish papules spread on a brown lichenoid patch over the left chest area 3 months after radiation therapy for an invasive carcinoma of the left breast. These lesions were confined to the radiation therapy site. Almost any type of irritant can provoke the isomorphic response including traumatisms, friction, infection and ultraviolet light. Only few cases of LP confined to radiation site have been described so far.
\end{abstract}

Key words: Lichen planus; Radiation therapy; Koebner phenomenon

\section{INTRODUCTION}

Lichen planus (LP) is an inflammatory skin condition characterized by the presence of pruritic, polygonal, purple flat-topped papules and plaques typically symmetrically distributed [1]. The response of Koebner is a common phenomenon of LP in areas prone to trauma or irritation [2]. This phenomenon occurs in multiple dermatoses including LP. However, only few cases of LP confined to radiation site have been described. Herein, we report a case of a radiation-induced LP.

\section{CASE REPORT}

We report the case of a 38-year-old diagnosed with a bifocal invasive ductal carcinoma of the left breast with left axillary lymph nodes damage. The patient underwent a radical left mastectomy with axillary node dissection. External radiation therapy (RT) of the left breast area and supraclavicular nodal area was performed. The dose given was 52,2 Gy in 1.6 Gy dose per session over 5 sessions/week for 6 weeks. Three months after the last session of RT, she presented to our department with multiple pruritic purplish papules spread on a brown lichenoid patch over the left chest area, limited to the radiation site (Fig. 1). The contralateral side, the rest of skin, mucous and nails were free of any skin lesion. The histological findings confirmed the diagnosis of LP (Fig. 2). Further investigations excluded any use of medications or other trigger factor and serology for hepatitis $\mathrm{B}$ and $\mathrm{C}$ were negative, leading to the diagnosis of radiation-induced LP. The lesions resolved gradually after 3 months of treatment with topical betamethasone.

Prior to the study, patient gave written consent to the examination and biopsy after having been informed about the procedure.

\section{DISCUSSION}

Lichen planus (LP) is an inflammatory dermatosis touching the skin and mucosae. It affects 0.5 to $1 \%$ of the population. Clinically it is characterized by pruritic violaceous polygonal papules with a shiny surface [1].

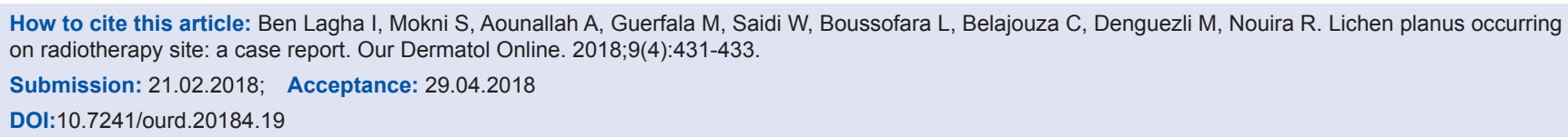




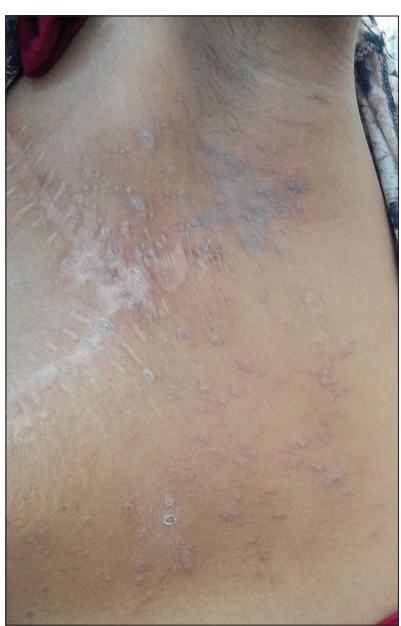

Figure 1: Hyperpigmented papules and plaques on a brown lichenoid patch located on the left chest radiated area.

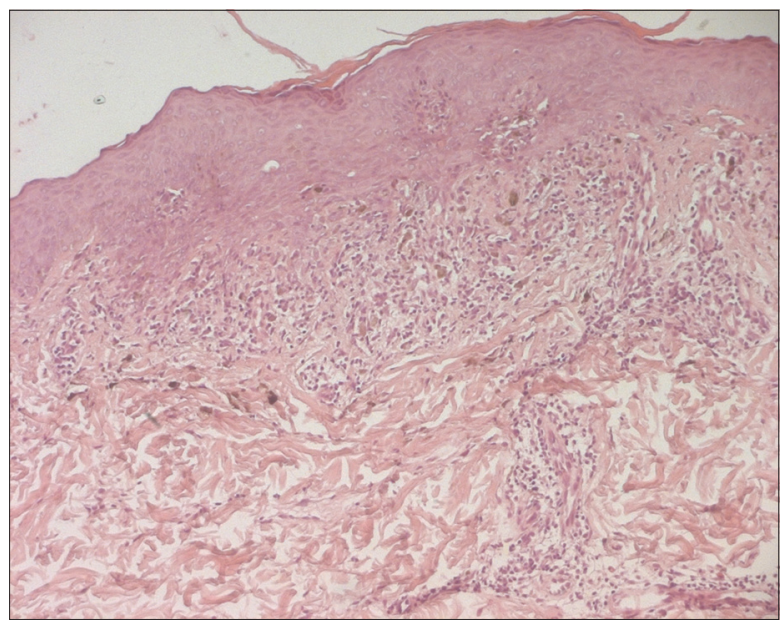

Figure 2: Histopathology: HE x 100: The epidermis showed mild acanthosis with basal vacuolar change. Ocassional civatte bodies are present in the basal layer. The papillary dermis showed a band like infiltrate of lymphocytes with exocytosis. There is prominent melanin incontinence.

Only a few cases of localized or generalized cutaneous LP occurring or re-appearing under radiation therapy have been reported [2-7]. Six cases were described to be confined to radiation field. While in other two cases the lesions were spread over the entire body. It has been suggested that an autoimmune process in which the damaged keratinocytes promote the elaboration of cytokines and therefore the activation of lymphocytes, causing the disease [3]. Other authors attributed the occurrence of lesson an isomorphic or Koebner response due to radiation injury [2,3]. The isomorphic response of Koebner occurs regularly in LP. Almost any type of irritation can provoke the isomorphic response including traumatisms, friction, infection and ultraviolet light. Koebnerization generally occurs within weeks of the trauma [6]. Shurman D and al. proposed the term "isoradiotopic response" to characterizethe phenomenon of secondary dermatoses occurring on radiationsites and to distinguish it from Koebner phenomenon. In fact, they noted two different points between the two phenomena: the variable time interval before eruption ranging from a few weeks to many years and the fact that other dermatoses not known to be involved in Koebner's phenomenon have been reported to appear after radiation (comedonal acne, folliculitis, erythema multiforme, scleroderma) [2].

In most of the cases reported in the literature, there was no history of LPand the lesions appeared for the first time after RT. The total given dose of radiation ranged from 18 Gy 7 to 60Gy 5 with an average of 46Gy. In majority of the cases, the lesions appeared within 1 to 3 months after treatment $[2,4,5,7]$. In one case of generalized generalized lichen ruber planus, the lesions appeared during the RT. In another case of lichen planopilaris after brain irradiation, the delay was about 10 years [6].

In our case, the radiotherapy induced the appearance of the LP lesions with a 3-month latency period from the moment radiotherapy was completed to the appearance of LP. We suggest the external radiation therapy to be considered as a cause of koebnerization in our patient. Reporting more similar cases could be helpful to determine if there are factors associated with an increased risk of developing LP after RT, such as the total given dose, treatment period and modalities.

\section{CONCLUSION}

Only a few cases of lichen planus provoked by radiotherapy have been reported. We present one more case of LP strictly restricted to radiation site therapy.

\section{Consent}

The examination of the patient was conducted according to the Declaration of Helsinki principles.

\section{REFERENCES}

1. Weston G, Payette M. Update on lichen planus and its clinical variants. Int J Womens Dermatol. 2015;1:140-9.

2. Shurman D, Reich HL, James WD. Lichen planus confined to a radiation field: The "isoradiotopic" response. J Am Acad Dermatol. 2004;50:482-3.

3. Vergilis-Kalner IJ, Sharma V, Sethi A. Lichen Planus Arising in Radiation Therapy Treatment Sites. Cutis. 2008;82:353-5.

4. Pretel M, Espana A. Lichen planus induced by radiotherapy. Clin Exp Dermatol. 2007;32:582-3.

5. Sajgane AA, Dongre AM. Radiotherapy-induced koebnerization in 
www.odermatol.com

lichen planus. Indian J Dermatol Venereol Leprol. 2012;78:665.

6. Perrin AJ, Donovan JC. Lichen planopilaris following whole brain irradiation. Int J Dermatol. 2014;53:468-70.

7. Morar N, Francis ND. Generalized lichen planus induced by radiotherapy: shared molecular mechanisms? Clin Exp Dermatol. 2009;34:434-5.
Copyright by Imene Ben Lagha, et al. This is an open-access article distributed under the terms of the Creative Commons Attribution License, which permits unrestricted use, distribution, and reproduction in any medium, provided the original author and source are credited.

Source of Support: Nil, Conflict of Interest: None declared. 